\title{
Helminth-Derived Product(S): Source for Potential Therapeutic
}

\author{
Azizul Haque \\ Department of Microbiology and Immunology \\ Geisel School of Medicine at Dartmouth, Lebanon \\ NH 03756, USA \\ Una Glamočlija \\ Genetics and Bioengineering \\ International University of Sarajevo \\ Hrasnička cesta 15, 71210 Sarajevo, BIH
}

\author{
Betul Akcesme \\ Genetics and Bioengineering \\ International University of Sarajevo \\ Hrasnička cesta 15, 71210 Sarajevo, BIH
}

\author{
Jasminka Mujić \\ Genetics and Bioengineering \\ International University of Sarajevo \\ Hrasnička cesta 15, 71210 Sarajevo, BIH
}

\begin{abstract}
Helminth parasites that inhabit mammalian body surfaces have a highly evolved relationship with the immune system. Many of these resident helminths carry out functions to ensure their survival in the hosts. To attain this objective helminth parasites adopt immunoregulatory mechanisms to counter host's hostile immune response. Indeed, immunomodulatory molecules have been discovered in the worm's extracts and in their excretion/secretion. In this review, we discuss the state of our understanding of the interplay between helminths and immune pathways. We also highlight the key challenges that must be confronted in identification of the helminth-derived molecules involved in immune modulation. We consider whether helminth-derived signaling hold promise for the design of novel therapeutic approaches for the treatment of inflammatory disorders (inflammatory bowel disease, allergies, and autoimmune diseases).
\end{abstract}

\section{INTRODUCTION}

Parasites and bacteria produce a wide range of molecules that can modulate eukaryotic immune responses. These include the bacterial enterotoxins, parasite-derived excretory-secretory products. These molecules can subvert protective mechanisms in order to facilitate pathogen colonization and persistence. In many instances they are able to inhibit inflammatory responses. In fact, there are examples of immune modulators that can exert either stimulatory or suppressive effects depending on the molecular nature, mode of delivery, timing and dose of exposure to the agents as well as characterization of the modes of action so that synthetic analogues that mimic the effects can be generated. There is presently great interest in the therapeutic exploitation of these factors, for example as a means to stimulate enhanced immune responses to a new generation of subunit vaccines or to inhibit deleterious immune mediated diseases [1,2].

Exposure to commensal and pathogenic microorganisms strongly influences our immune system. Exposure to parasites and bacteria was frequent before humans constructed their current highly hygienic environment. Today, in highly industrialized countries, the contact between humans and the microorganism is dramatically diminishing. Congruent with the decline in helminthic and bacterial infections is an increase in the prevalence of autoimmune and inflammatory diseases. Incidence of some of these autoimmune and inflammatory diseases is now on the rise in the developing countries that are making rapid economic growth as people are adapting to more hygienic life-style due to affluence. It is possible that exclusion of many of the parasites and bacteria from the environment has permitted the emergence of immune-mediated diseases [3]. It is believed that parasites and humans have been co-evolved in the evolutionary history and the maintenance of an immunoregulatory environment may be promoted by parasites to ensure their survival in the hosts. A breakdown of this regulation that involves multiple levels of host regulatory cells and cytokines, is likely to 
result into pathological disease.

In this review we will highlight the process in which helminths modulate both the innate and the adaptive arms of the immune system. It is our view that identification of the microbes-derived molecules involved in immune modulation will pave the way to novel therapeutic approaches for the treatment of inflammatory diseases. Here, we provide an overview of the findings from animal models and clinical studies, and additionally explores the potential for translation of these findings to the clinic.

\section{BACKGROUND}

Intestinal helminth infections are of particular interest because they have strong immune regulatory effects on their host $[4,5]$. The most common helminth infections humans are caused by geohelminths (also known as intestinal and soil-transmitted helminths). Geohelminth parasites include Ascaris lumbricoides, Trichuris trichiura and hookworm (Ancylostoma duodenale and Necator americanus). Other important helminthic infections in humans include schistosomiasis, fascioliasis and filariasis, which are tissue-dwelling parasites.

Currently, there is much interest in whether helminthassociated immune regulation may ameliorate allergy and autoimmune diseases, with investigations in both laboratory models and human trials. Analyses have consistently revealed bioactive molecules in extracts of helminths or in their excretory/secretory products that modulate the immune response of the host. Here, we illustrate the range of immunomodulatory molecules in selected parasitic trematodes, cestodes and nematodes, their impact on the immune cells in the host and how the host may recognize these molecules. Several years ago, we demonstrated that treatment with the extracts of Dipetalonema vitae male worms (a filarial nematode parasite) resulted into immunosuppression thereby enhancing the parasitemia in infected animals [6]. Recently, a tegumental coat antigen was isolated from the cestode parasite Fasciola hepatica (FhTeg) that had shown suppressive effect in vivo by directly targeting dendritic cells, impairing their ability to drive Th1 responses. In addition, FhTeg inhibits the ability of mast cells to drive the Th1 immune response by suppressing cytokine secretion (TNF- $\alpha$, IL- 6 , IFN- $\gamma$, and IL-10) and ICAM1 expression in mast cells stimulated with LPS or heat-inactivated Bordetella pertussis Ag [7].

Symbiotic bacteria may be present in some nematode parasites. Lipopolysaccharide-like molecules have been detected in sterile products of filarial nematode Onchocerca volvulus stages, which could originate from Wolbachia bacteria related to Gram-negative Rickettsiales. Wolbachia bacteria are known to be abundant in the hypodermis and the female reproductive organs of $O$. volvulus. It has been demonstrated that monocyte/macrophage would be a major target cell for immunomodulatory parasite-derived and intraparasitic, bacteria-derived molecules, thereby contributing to the host's cellular hyporesponsiveness [8].

Treating animals with helminths (eggs, larvae, extracts) causes dampening, and in some cases prevention of allergic and autoimmune diseases. Clinical studies showed that administration of eggs of Trichuris suis (a parasitic nematode of swine) reduces the disease severity to patients with inflammatory bowel disease [9]. Trials using colonization of helminths like Necator americanus (hookworm) or $T$. suis (porcine whipworm) show that they are safe and may be effective therapies for the control of the aberrant intestinal inflammation seen in Crohn's disease and ulcerative colitis [10]. Treatment with Schistosoma mansoni and Ancylostoma caninum soluble proteins significantly suppressed the inflammation and ameliorated trinitrobenzene sulphate (TNSB)-induced colitis [11]. Similar inhibitory effect on intestinal inflammation was observed when TNSBinduced mice received larvae of Heligmosomoides polygyrus, a duodenal nematode parasite [12].

Of note, $T$. suis when used in a randomized, doubleblind, placebo-controlled clinical trial in Denmark against allergic rhinitis, no therapeutic effect was seen [13]. This raises the question whether the effectiveness of helminth therapy, especially with live intestinal nematodes will be disease specific, and may not be efficient enough against all inflammatory diseases. Further, such variation may be related to host's genetic or to environmental factors including the composition of bacterial population that colonizes the host's gut.

Multiple sclerosis (MS) is an inflammatory autoimmune demyelinating disease affecting the Central Nervous System (CNS), in which Th1 and Th17 cells appear to recognize and react against certain myelin sheath components [14,15]. Mucosal tolerance has been considered a potentially important pathway for the treatment of autoimmune disease, including MS and experimental conditions such as experimental autoimmune encephalomyelitis (EAE). It has been demonstrated that treatment with soluble products from the nematodes $T$. suis and Trichinella spiralis induces significant suppression of symptoms in EAE, a validated animal model for multiple sclerosis [16]. This amelioration in EAE pathogenesis is associated with the inhibition of TNF- $\alpha$ and IL-12 secretion by TLRactivated human DCs. Furthermore, helminth-primed human DCs differentially suppress the development of Th1 and/or Th17 cells [17].

The incidence of type 1 diabetes (TID) is increasing dramatically in the developed world. While there may be several reasons for this, improved hygienic conditions and public health measures have impacted our 
interactions with certain infectious microorganisms, especially with helminths. Therapeutic potential of both live infections as well as helminth-derived products has been explored in the animal models of TID [18]. It was shown that infection with Schistosoma mansoni could prevent diabetes onset in NOD mice [19]. Other studies confirmed the potent effect of other helminth infections on T1D in NOD mice including Trichinella spiralis, Heligmosomoides polygyrus [20,21]. Protection was associated with an augmented Th2 response, increased generation of regulatory $\mathrm{T}$ (Treg) cells, production of IL10 by $\mathrm{T}$ and $\mathrm{B}$ cells and reduction in infiltrating $\mathrm{CD} 8^{+} \mathrm{T}$ cells in the pancreas; all of which would have the potential to inhibit diabetes onset. Interestingly, $H$. polygyrus infection induces non-regulatory $\mathrm{T}$ cells that produce IL-10 independently of STAT6 signaling. This signifies deficiency of $\mathrm{Th} 2$ response, and in this Th2 deficient environment $\mathrm{IL}-10$ is essential for T1D inhibition [21]. The use of defined parasite products to influence diabetes onset will provide an additional way of addressing mechanisms of diabetes prevention.

A number of studies have now investigated the immunomodulatory mechanisms of helminth-derived soluble molecules in controlling allergy. [3,22]. As earlier as 1983, we tested the hypothesis whether Filarial nematode $D$. Viteae could produce substances that might down-regulate hosts' allergic responses [23]. In this report, we described that a dialyzable fraction of $D$. vitae male worms' released product inhibited passive cutaneous anaphylaxis (PCA, anti-ovalbumin and ovalbumin). In subsequent studies other researchers described the immunomodulatory activity of ES-62, one of the excretory/secretory products of the filarial worm, Acanthocheilonema viteae (some scientists prefer to term Dipetalonema viteae as Acanthocheilonema viteae). Molecular characterization has shown that ES-62 is a 62$\mathrm{kDa}$ phosphorylcholine (PC)-containing glycoprotein. ES-62-treated mice were protected in a cutaneous model of immediate-type hypersensitivity induced by oxazolone [24]. This ES-62 product has been shown to interact with a variety of cells of the immune system including $B$ and $\mathrm{T}$ lymphocytes, dendritic cells, macrophages and mast cells. It appears that that ES-62 acts on $\mathrm{T}_{\mathrm{H}} 17$ responses rather than $\mathrm{T}_{\mathrm{H}} 1$ responses [25]. It has been shown that mice infected with nematode parasite, $H$. polygyrus are protected from allergic airway inflammation. Further, when the excretory-secretory material from this parasite was added to sensitizing doses of ovalbumin, the subsequent allergic airway response was suppressed, and this condition was associated with a lower ratio of effector [CD4 (+) CD25 (+) Foxp3 (-)] to regulatory [CD4 (+) Foxp3 (+)] T (Treg) cells, and reduced Th1, Th2 and Th17 cytokine production [26]. Of note, allergic asthma is less prevalent in countries with parasitic helminth infections, it appears that helminth-derived molecules can divert the immune system towards an anti- inflammatory phenotype and may have therapeutic potential in inflammatory diseases.

\section{MECHANISMS OF ACTION}

Evidence from human studies and mouse models shows that infection with parasitic helminths has a suppressive effect on the pathogenesis of some inflammatory diseases $[1,5]$. The overall paradigm in the way the helminth-derived products mediate immunomodulation appears to demonstrate their ability to impact the various immune activation pathways leading to a reduction in inflammation. Some helminthes and their products inhibit inflammatory responses through effects on both the innate and adaptive immune response (Fig.1). Helminth suppression of immunopathology generally involves $\mathrm{CD}^{+}{ }^{+}$regulatory $\mathrm{T}$ cells (Tregs, either Foxp3 ${ }^{+}$ or Foxp $3^{-}$), IL-4-responsive cells, and TGF- $\beta$, IL-10 and $\mathrm{Th} 2$ cytokines. The potential regulatory role of $\mathrm{CD}^{+}$ Tregs, regulatory $\mathrm{B}$ cells has not yet been clearly defined.

The favored mechanism is that microbial and parasitederived products interact directly with pathogen recognition receptors to subvert proinflammatory signaling via $\mathrm{T}$ regulatory cells (Tregs), thereby inducing anti-inflammatory effects and control of autoimmune disease. An important immunoregulatory cytokine that participate in inducing tolerance is IL-10. This cytokine is produced by Foxp3 positive Treg cells (e.g. Tr1) [27, 28]. Although this may suggest that $\operatorname{Tr} 1$ cells are developmentally related to Treg cells, an alternative possibility is that IL-10 gene induction does not require Foxp3 expression [29]. One of the important components in immune activation implicates the role of $\mathrm{TH} 17$ subtype helper cells that secret IL-17 cytokine [30]. Coexpression of T-bet and ROR $\gamma \mathrm{t}$, which is found in subsets of IL-17-producing T-helper cells, may be an evolutionarily conserved transcriptional program that originally developed as part of the innate defense against infections but that also confers an increased risk of immune-mediated pathology [31]. The relationship between the various effector (Th1, Th17) and regulatory (Treg, Tr1) $\mathrm{T}$ cell subsets still needs to be clarified, in terms of antigen-specificity, development, and function. It seems that helminth-derive substance(s) may have impact on the innate immunity by targeting dendritic cells (DCs) and by regulating TLR signaling pathway. They may also down-regulate the Th1 response and polarize hosts' response towards Th2 response (Fig.1). By balancing these responses, helminths product(s) can reduce inflammation in autoimmunity and in other inflammatory disorders.

One approach would be to identify and isolate helminthderived molecules that will facilitate iTreg cell induction. Another possibility would be ex vivo expansion and reinfusion of Treg cells, a strategy being attempted for 
the treatment of autoimmune diabetes [32, 33]. Specifically expanding Treg cells might facilitate Treg cell localization to the gut and enhance suppressive activity. Thus, understanding the mechanisms of immune homeostasis to the intestine may provide insight into the pathogenesis and treatment of inflammatory bowel disease, colitis, and diabetes type 1 . This may also apply to inhibiting Multiple Sclerosis pathogenesis as it has previously been shown that suppression of IL-17 and enhancement of Treg response are involved in amelioration of EAE pathogenesis $[14,15]$. As regards to inhibiting allergy/asthma, it is likely that helminthderived molecules will inhibit mast cell activation induced by high affinity $\mathrm{IgE}$ receptor FceRI, a critical receptor in patients with $\mathrm{IgE}$-mediated allergy/asthma.

\section{CONCLUSION AND FUTURE DIRECTION}

Helminthic worms have evolved strategies to manipulate the host immune system, some of which may lead to a reduction in inflammation. Characterization of the ways in which these organisms mediate an anti-inflammatory response and identification of parasite-derived molecules involved in immune modulation will contribute to the discovery of novel therapeutic approaches for the treatment of inflammatory disease.

Although helminth infections are implicated in protecting the host against immune-mediated diseases, they are also believed to cause significant morbidity and in some cases mortality in endemic areas through their effects on nutrition, growth and cognition; and they have deleterious effects on vaccine immune responses. Thus, it might be more practical to focus on individual helminth-derived immunomodulatory molecules to selectively induce regulatory immune responses (particularly those expressed during the chronic phase of infection) and avoid any possible side effects of natural worm infections. The introduction of live helminths in intestine may favor the outgrowth or suppression of certain bacteria in the gut, which in turn can impact host's immunity. Hence, the priority would be to focus on the molecules released by helminth parasites (as excretory-secretory products). Clearly, further progress needs to be made in identifying parasite derived molecules, the ways in which they interact with the immune system and how they mediate immunomodulation in the genetically heterogeneous human population.

Much remains to be resolved regarding identification of potential helminth-derived biomodulators, timing and dose of exposure to the agents as well as characterization of the modes of action so that synthetic analogues that mimic the effects can be generated. Analyses have consistently revealed bioactive molecules in extracts of helminths or in their excretory/secretory products that modulate the immune response of the host. It is our view that parasitic helminths are an untapped source of immunomodulatory substances that, in pure form, could become new drugs (or models for drug design) to treat disease. There are many examples of the partial characterization of helminth-derived immunomodulatory molecules, but these have not yet translated into new drugs, reflecting the difficulty of isolating and fully characterizing proteins, glycoproteins and lipid-based molecules from small amounts of parasite material. However, this should not deter the investigator, since analytical techniques are now being used to accrue considerable structural information on parasite-derived molecules, even when only minute quantities of tissue are available. With the introduction of methodologies to purify and structurally-characterize molecules from small amounts of tissue and the application of high throughput immunological assays, one would predict that an assessment of parasitic helminths will yield a variety of novel drug candidates in the coming years.

In order to develop non-living parasite-derived therapeutic agents, the molecular motifs that are responsible for protection from immunopathology must first be identified, their cellular targets need to be characterized and gene families implicated in immunomodulation have to be defined. A few molecules have already been identified as potential candidates for immunotherapy. It is important that efforts continue to bring these molecules to the clinical setting as they hold potential for human therapy of immunopathological conditions.

\section{APPENDICES}

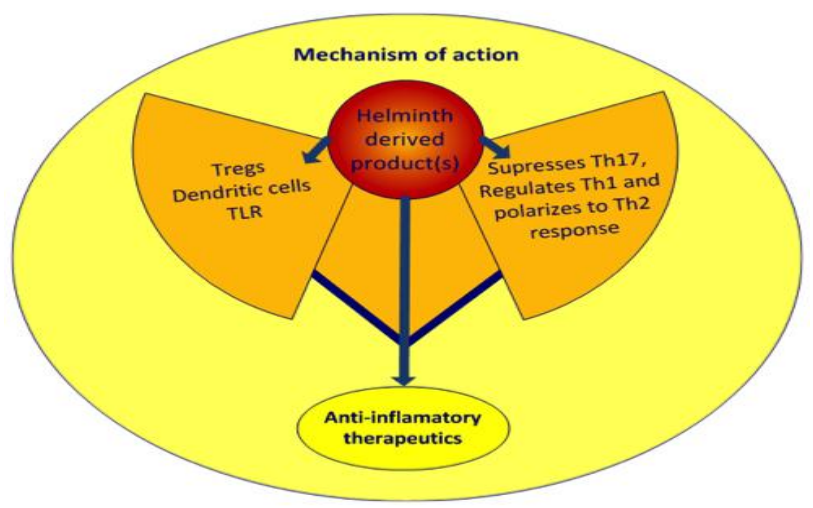

Figure 1 - Helminth-derived product(s) are potential candidates for immunotherapy. These molecules mediate suppression through $\mathrm{CD}^{+}$regulatory T cells (Tregs, either Foxp $^{+}$or Foxp $3^{-}$), modulatory dendritic cells (DCs), and by impacting TLR signaling. Regulatory responses induced by helminth-derived substances include suppression of Th17, Th1 response and polarization to Th2 response. Engagement of TLR on DCs may determine the Th2 polarization of the T cells. 


\section{REFERENCES}

[1] Y. Osada and T. Kanazawa, Parasitic helminthes: new weapons against immunological disorders. J. Biomed. Biotech. Article ID 743758, 1-9, 2010.

[2] D.E. Elliott and J.V. Weinstock, Helminth-host immunological interactions: prevention and control of immune-mediated diseases. Ann NY Acad Sci 1247: 83-96, 2012.

[3] H.J. McSorley et al., Immunomodulation by helminth parasites: defining mechanisms and mediators. Int $\mathrm{J}$ Parasitol. 43:301-310, 2013.

[4] M.H. Jouvin and J.P. Kinet, 2012. Trichuris suis ova: testing a helminth-based therapy as an extension of the hygiene hypothesis. J Allergy Clin Immunol 130:3-10, 2012.

[5] J.V. Weinstock, The worm returns. Nature 491: 183-185, 2012.

[6] A. Haque et al., Dipetalonema viteae infection in hamsters: enhancement and suppression of microfilaraemia. Parasitology 76, 77-84, 1978.

[7] K.V. Vukman et al., Fasciola hepatica tegumental coat impairs mast cells' ability to drive Th1 immune responses. J Immunol. 190:2873-2879, 2013.

[8] M.J. Taylor et al., Wolbachia filarial interactions. Cell Microbiol Dec 4. doi: 10.1111/cmi.12084, 2012.

[9] R.W. Summers et al., Trichuris suis seems to be safe and possibly effective in the treatment of inflammatory bowel disease. Am J Gastroenterol. 98:2034-2041, 2003.

[10] D.E. Elliott and J.V. Weinstock, Where are we on worms. Curr Opin Gastroenterol. 28:551-556, 2012.

[11] N.E. Ruyssers et al., Therapeutic potential of helminth soluble proteins in TNBS-induced colitis in mice. Inflamm Bowel Dis. 15:491-500, 2009.

[12] T.L. Sutton et al., Anti-Inflammatory mechanisms of enteric Heligmosomoides polygyrus infection against trinitrobenzene sulfonic acid-induced colitis in a murine model. Infect Immun. Oct;76(10):4772-4782, 2008.

[13] P. Bager et al., Helminth infection does not reduce risk for chronic inflammatory disease in a population-based cohort study. Gastroenterology 142:55-62, 2012.

[14] A. Rostami and B. Ciric, Role of Th17 cells in the pathogenesis of CNS inflammatory demyelination. J Neurol Sci. doi:pii: S0022-510X(13)00108-1. 10.1016/j.jns.2013.03.002, 2013.

[15] S. Begum-Haque et al., Glatiramer acetate biases dendritic cells towards an anti-inflammatory phenotype by modulating OPN, IL-17, and ROR $\gamma$ t responses and by increasing IL-10 production in experimental allergic encephalomyelitis. J Neuroimmunol. 254:117-1124, 2013.

[16] J. Correale and M. Farez, Helminth antigens modulate immune responses in cells from multiple sclerosis patients through TLR2-dependent mechanisms. J Immunol. 183:5999-6012, 2009.

[17] L. M. Kuijk et al., Soluble helminth products suppress clinical signs in murine experimental autoimmune encephalomyelitis and differentially modulate human dendritic cell activation. Mol Immunol. 51:210-218, 2012. of Type 1 diabetes (T1D). Int J Parasitol. 43:311-8, 2013.

[19] P. Zaccone et al., Immune modulation by Schistosoma mansoni antigens in NOD mice: effects on both innate and adaptive immune systems. J Biomed Biotechnol. 2010:795210. doi: 10.1155/2010/795210, 2010.

[20] K.A. Saunders et al., Inhibition of autoimmune type 1 diabetes by gastrointestinal helminth infection. Infect Immun. 75:397-407, 2007.

[21] P.K. Mishra et al., Prevention of type 1 diabetes through infection with an intestinal nematode parasite requires IL-10 in the absence of a Th2-type response. Mucosal Immunol. 6:297-308, 2013.

[22] D.B. Shor et al., The hygiene theory harnessing Helminthes and their Ova to treat autoimmunity. Clinic Rev Allerg Immunol DOI 10.1007/s12016-012-8352-8359, 2013.

[23] A. Haque et al. Immune unresponsiveness and its possible relation to filarial disease. Contribution to Microbiology and Immunology 7: 9-21, 1983.

[24] M.M. Harnett et al., The phosphorycholine moiety of the fimarial nematode immunomodulator ES-62 is responsible for its anti-inflammatory action in arthritis. Ann Rheum Dis 67:518-523, 2008.

[25] M.M. Harnett et al., The therapeutic potential of the filarial nematode-derived immunomodulator, ES-62 in inflammatory disease Clin Exp Immunol 159: 256-267, 2010.

[26] H.J. McSorley et al., Suppression of type 2 immunity and allergic airway inflammation by secreted products of the helminth Heligmosomoides polygyrus. Eur J Immunol. 42:2667-2682, 2012.

[27] A.Y. Rudensky, Regulatory T cells and Foxp3. Immunol Rev. 241:260-268, 2011.

[28] J.R. Grainger et al., Helminth secretions induce de novo T cell Foxp3 expression and regulatory function through the TGF- $\beta$ pathway. J Exp Med. 207:2331-2341, 2010.

[29] S. Sakaguchi et al., The plasticity and stability of regulatory T cells. Nat Rev Immunol. doi: 10.1038/nri3464, 2013.

[30] S. Huber et al., Th17 cells express interleukin-10 receptor and are controlled by Foxp $3^{-}$and Foxp3+ regulatory CD4+ $\mathrm{T}$ cells in an interleukin-10-dependent manner. Immunity 34:554-565, 2011.

[31] C.S. Klose et al., A T-bet gradient controls the fate and function of CCR6-ROR $\gamma \mathrm{t}+$ innate lymphoid cells. Nature 494:261-265, 2013.

[32] N. Marek-Trzonkowska et al., Clinical application of regulatory T cells in type 1 diabetes. Pediatr Diabetes. doi: 10.1111/pedi.12029, 2013.

[33] S.M. Cabrera et al., Targeting regulatory T cells in the treatment of type 1 diabetes mellitus. Curr Mol Med. 12:1261-1272, 2012. 